\title{
Loco-Regional Treatment for Intact Primary Tumor in Patient with De Novo Metastatic Breast Cancer; Comments and Concerns of ECOG-ACRIN 2108 Trial
}

\author{
Atilla Soran (D), Serdar Özbaş (D), Lütfi Doğan (D), Efe Sezgin (D), Vahit Özmen (D), Sushil Beriwal (D), Adam Brufsky (iD \\ On behalf of The Breast Disease Working Group
}

Cite this article as: Soran A, Özbaş S, Doğan L, Sezgin E, Özmen V, Beriwal S, Brufsky A. Loco-Regional Treatment for Intact Primary Tumor in Patient with De Novo Metastatic Breast Cancer; Comments and Concerns of ECOG-ACRIN 2108 Trial. Eur J Breast Health 2020; 16(3): 158-159.

Khan et al. (1) presented the results of the multicenter, phase 3, ECOG ACRIN 2108 study at the plenary session of ASCO 2020 virtual meeting earlier than expected. ECOG-ACRIN 2108 (Eastern Cooperative Oncology Group-American College of Radiology Imaging Network - NCT01242800) trial recruited 390 women with de novo metastatic breast cancer (MBC) from February 2011 through July 2015 to determine whether the addition of locoregional treatment improved overall survival (OS). While 134 of these cases were excluded from the study for different reasons, 256 eligible patients were assigned to systemic therapy based on patient and tumor characteristics. Those who did not progress during 4 to 8 months of treatment were then assigned to continue systemic therapy alone (ST - n=131) or ST plus locoregional treatment with surgery $+/$ - radiation (LRT $-\mathrm{n}=125$ ).

The primary endpoint of the study was overall survival, and secondary endpoints were the time for locoregional progression and healthrelated quality of life measurement (HRQoL). There was no significant difference between the groups in terms of patients' age, race, menopausal status, tumor burden, hormone receptor and HER2 status. The median patient age was approximately 56 years, and nearly two thirds of patients were postmenopausal. Surgery was done in 109 (86\%) of 125 patients in the early LRT group. Only 87 (80\%) of these patients had tumor free surgical margins and 74 patients $(68 \%)$ received locoregional radiotherapy. On the other hand, 25 of the 131 patients in the ST group (19\%) received palliative surgery during the study.

They found no statistical difference between the two groups in terms of 3-year OS (68.4\% vs 67.9\%) (HR, 1.09; 90\% CI, 0.80-1.49; $\mathrm{p}=0.63$ ). At an average of 53 months follow up; the average OS for the entire study population was 54 months.

In addition, no progression-free survival benefit was observed between the ST and early LRT groups ( $\mathrm{p}=0.40$ ). However, locoregional recurrence or progression was significantly higher in the ST arm alone (25.6\% vs $10.2 \%$; $=0.003)$.

When subgroup analyzes were performed; according to the tumor molecular subtypes (HER2-positive tumor, hormone receptor-positive and HER2-negative tumor) no significant difference was observed between ST and early LRT study groups in terms of OS.

Regarding HRQoL measurements, the percentages of patients who completed the FACT-B questionnaire at the 6., 18. and 30. months following randomization were $81 \%, 60 \%$ and $51 \%$, respectively. Although there was no significant difference in HRQoL measurements between both groups at 6 and 30 month post randomization; it was worse in the early LRT arm at 18 month post randomization.

As conclusion, Dr. Khan et al. (1) stated that based on the available data, patients with de novo MBC should not be offered locoregional therapy for primary tumor with the expectation of survival benefit. First of all, because of some missing details in the presentation, it is important to wait for the publication of this study to make a better assessment and to reach some definite conclusions. However, we want to share our comments and concerns on this study under the following headings:

- While planning the study statistics, 3-year overall survival was predicted as 30\% in the ST group and 49.3\% in the early LRT group, but in the follow-up 3-year survival was $67.9 \%$ in the ST group and $68.4 \%$ in the early LRT group (HR, 1.09; 90\% CI, 0.80-1.49; 
$\mathrm{p}=0.63)$. High survival rate in this control group results decreases power of the study significantly. Although 125 patients were randomized to the early LRT group; only 109 were treated surgically which further decreases power of the study to detect the difference in survival.

- Multivariate analysis is also missing.

- Although one of the inclusion criteria for randomization is "complete resection with tumor free surgical margins"; it was achieved in $87 / 109$ patients and the surgical margin remained positive in approximately $20 \%$ of the early LRT group. Besides details about extent of surgery for axilla and radiation volumes are not available.

- The rate of patients with skin invasion, presence of satellite nodules and fascia invasion appears to be quite high in the study ( $44 \%$ of patients have skin involvement, skin nodules and fascia invasion; $48 \%$ of them have T4 and / or N2 / 3 diseases). The distribution of these patients with locally advanced disease is not specified.

- Subgroup analysis according to the metastatic site is missing. Although, $38 \%$ of patients had only bone metastases, a subgroup analysis for this group was not performed. Similarly, no organspecific comparison was made between the groups.

- Although the initial and final evaluation comparisons of HRQoL measurements are similar; Considering significant locoregional progression in patients with metastasis, we think it would be important to evaluate the long-term or 3-year HRQoL score.

- The patients with 0 months follow-up were included in the analysis, and when the deaths within 6 months were examined, it was seen that the mortality rates in the early LRT arm were high. The importance of surgery for survival can be evaluated with an analysis to be made by subtracting early deaths from statistical evaluation, and then analysis of those living more than 3 years and more.
We will get answers to some of the questions mentioned above after the publication of the manuscript. It seems that the ECOG-ACRIN 2108 study may not powered to answer the question of whether primary surgery provides a survival advantage in de novo MBC. We will continue to look forward to the results of the JCOG 1017 trial and Stereotactic body radiation therapy studies.

However, ECOG-ACRIN 2108 study gives important data in terms of local control. It is important to achieve local control in oligometastatic cases, especially in patients with bone only metastases. In addition, surgical removal of the primary tumor is important to prevent local spread to the pleura and pericardium. In order to determine who will benefit from early surgery in de novo MBC patients, we need more studies and information in terms of tumor and patient characteristics including biomarkers.

Therefore, regarding the presented data concluding that early surgery has no place in de novo MBC patient's treatment is eliminating the possibility of long-term no evidence of disease or cure. Loco-regional treatment for intact primary tumor for de novo MBC need to be considered case by case with input and discussions from all stake holders.

\section{Reference}

1. Khan SA, Zhao F, Solin LJ, Goldstein LJ, Cella D, Basik M, Golshan M, Julian TB, Pockaj BA, Lee CA, Razaq W, Sparano JA, Babiera GV, Dy IA, Jain S, Silverman P, Fisher C, Tevaarwerk AJ, Wagner LI, Sledge GW. A randomized phase III trial of systemic therapy plus early local therapy versus systemic therapy alone in women with de novo stage IV breast cancer: A trial of the ECOG-ACRIN Research Group (E2108). J Clin Oncol 2020; 38(Suppl): pp.LBA2. [Crossref] 